\title{
Characterizing Exon Skipping Efficiency in DMD Patient Samples in Clinical Trials of Antisense Oligonucleotides
}

\author{
Joel Z. Nordin ${ }^{*}{ }^{1}$, Yoshitaka Mizobe ${ }^{*}, 1$, Harumasa Nakamura ${ }^{2}$, Hirofumi Komaki ${ }^{3}$, Shin'ichi Takeda ${ }^{1}$, Yoshitsugu Aoki ${ }^{1}$ \\ ${ }^{1}$ Department of Molecular Therapy, National Institute of Neuroscience, National Center of Neurology and Psychiatry ${ }^{2}$ Clinical Research Support Office, \\ Translational Medical Center, National Center of Neurology and Psychiatry ${ }^{3}$ Department of Child Neurology, National Center Hospital, National Center of \\ Neurology and Psychiatry \\ *These authors contributed equally
}

\section{Corresponding Author}

Yoshitsugu Aoki

tsugu56@ncnp.go.jp

\section{Citation}

Nordin, J.Z., Mizobe, Y.,

Nakamura, H., Komaki, H., Takeda, S.,

Aoki, Y. Characterizing Exon Skipping

Efficiency in DMD Patient Samples

in Clinical Trials of Antisense

Oligonucleotides. J. Vis. Exp. (159),

e60672, doi:10.3791/60672 (2020).

\section{Date Published}

May 7, 2020

DOI

$10.3791 / 60672$

URL

jove.com/video/60672

\section{Abstract}

Duchenne muscular dystrophy (DMD) is a degenerative muscle disease that causes progressive loss of muscle mass, leading to premature death. The mutations often cause a distorted reading frame and premature stop codons, resulting in an almost total lack of dystrophin protein. The reading frame can be corrected using antisense oligonucleotides (AONs) that induce exon skipping. The morpholino AON viltolarsen (code name: NS-065/NCNP-01) has been shown to induce exon 53 skipping, restoring the reading frame for patients with exon 52 deletions. We recently administered NS-065/NCNP-01 intravenously to DMD patients in an exploratory investigatorinitiated, first-in-human trial of NS-065/NCNP-01. In this methods article, we present the molecular characterization of dystrophin expression using Sanger sequencing, RT-PCR, and western blotting in the clinical trial. The characterization of dystrophin expression was fundamental in the study for showing the efficacy since no functional outcome tests were performed.

\section{Introduction}

Duchenne muscular dystrophy (DMD) is a degenerative muscle disease causing progressive loss of muscle mass, respiratory failure, and cardiomyopathy, and it leads to premature death ${ }^{1}$. The disease is caused by a lack of the large structural muscle protein dystrophin ${ }^{2}$. Mutations in the $\mathrm{DMD}$-gene on the $\mathrm{X}$-chromosome are recessive, and the disease affects 1 in 3500-5000 new-born males ${ }^{3,4,5}$.
The mutations are often large deletions in a hotspot region between exons 44 and 55 that lead to a distorted reading frame and premature stop codons, causing nonsensemediated decay and an almost total lack of dystrophin protein $^{6,7,8}$. The reading frame can be corrected using antisense oligonucleotides (AONs) that induce exon skipping and restore the reading frame, partially restoring dystrophin 
expression and delaying disease progression $9,10,11$. The morpholino AON eteplirsen, which was recently approved by the Federal Drug Agency (FDA), induces skipping of exon 51 and can restore the reading frame in patients with exon 52 deletions $^{12,13}$. However, exon 53 skipping restores the reading frame for patients with exon 52 deletions, and it can potentially treat approximately $10 \%$ of DMD patients ${ }^{14}$. The morpholino AON drug NS-065/NCNP-01 has been shown to induce exon 53 skipping in human cells, and we recently administered NS-065/NCNP-01 to DMD patients in a phase 1 open-label dose-escalation clinical trial (hereinafter, referred to as "the study") (registered as UMIN: 000010964 and ClinicalTrials.gov: NCT02081625) ${ }^{14}$. The study showed a dose-dependent increase of exon 53 skipping based on RT-PCR and dystrophin protein levels based on western blotting, and no severe adverse drug events or dropouts were observed $^{14}$.

In all clinical trials, the analysis of the results are of paramount importance. For DMD clinical trials, a debate is still ongoing regarding the best method to show a treatment benefit. Clinical tests such as the 6-minute walk test have certain drawbacks. Molecular characterization of the dystrophin expression can be performed using several methods, such as RT-PCR, qPCR, digital-PCR, western blotting, and immunohistochemistry. However, the extent of protein expression restoration that is required to impart a clinical benefit remains unclear. In this methods article, we describe in detail the RT-PCR and western blotting methods used to determine the exon skipping and protein levels, respectively, in the phase 1 trial of the AON skipping drug NS-065/NCNP-0 $01^{14}$.

\section{Protocol}

The operational procedure for the investigator-initiated trial has been approved by the NCNP ethical committee (approval ID: A2013-019).

\section{Preparation of muscle samples}

NOTE: Biceps brachii or quadriceps muscles are often selected as biopsy sites. However, the tibialis anterior was used in the clinical trial.

\section{Muscle biopsy from patients}

1. Mark the incision site before skin preparation.

2. Prepare saline-dampened gauze for the biopsy specimen. Squeeze the gauze well.

3. Inject local anaesthesia into skin and subcutaneous tissue, but not into muscle. Make sure of the depth of the anesthesia by the absence of cutaneous pain.

NOTE: General anaesthesia is generally used in paediatric patients under 15 years of age.

4. Make an incision from the skin to the subcutaneous tissue. Use small retractors to open the incision and separate the subcutaneous fat.

NOTE: At this step, a portion of the skin can be cut for fibroblast culture.

5. Make another small incision in the fascia and cut the fascia further. Clamp the edges using mosquito forceps to expose the muscle.

6. Use a suture to pull up the selected portion of the muscle. Make a small tunnel using Iris scissors and insert mosquito forceps into the tunnel. 
7. Separate the muscle bundle using forceps and cut both ends of the target portion.

NOTE: Muscle biopsy specimens should be around the size of a pencil for adult patients (length 1.0-1.5 $\mathrm{cm}$, diameter $0.8-1.0 \mathrm{~cm}$ ). The specimen should be around $1 \mathrm{~cm}$ in length and $5 \mathrm{~mm}$ in diameter in paediatric patients.

8. Wrap the specimen in saline-dampened gauze and transport the fresh muscle at room temperature (RT) immediately to a facility where the specimen can be prepared for further analysis.

NOTE: The specimens should be transported at $4{ }^{\circ} \mathrm{C}$ if the duration of transportation exceeds 1 hour.

\section{Muscle sample preparation}

1. Mix equal volumes of tragacanth gum and water until the gum becomes soft and sticky. Load the mixture into 25 $\mathrm{mL}$ syringes. The syringes can be stored in a refrigerator.

2. Place approximately 0.5 to $1 \mathrm{~cm}$ of tragacanth gum on cork discs. Label the discs on the opposite side.

3. Place a container of isopentane in liquid nitrogen until some of the liquid freezes.

4. Place the specimen in the tragacanth gum. The longitudinal axis of each muscle should be perpendicular to the cork. Place gum around the bottom of each muscle to help proper placement.

5. Using tweezers, place the muscle/cork specimen in cold isopentane prepared in step 2.3 to freeze. Move the specimen constantly for $1 \mathrm{~min}$ or until completely frozen, and place on dry ice temporarily.

6. Place the specimen in glass vials and store at $-80^{\circ} \mathrm{C}$.

\section{Muscle sectioning}

1. Set up the cryostat for sectioning with a working temperature of $-25^{\circ} \mathrm{C}$.

2. Mount the cork/muscle block and trim until flat sections are achieved.

3. Use a section thickness of $10 \mu \mathrm{m}$ for RT-PCR and western blotting. Use a thickness of 6-8 $\mu \mathrm{m}$ and 10-12 $\mu \mathrm{m}$ for immunohistochemistry or haematoxylin and eosin staining, respectively. Put sliced sections in $2.0 \mathrm{~mL}$ tubes and store at $-80^{\circ} \mathrm{C}$ for RT-PCR and western blotting.

\section{RNA extraction and reverse transcription polymerase chain reaction (RT-PCR)}

1. Extract approximately 10 slices with $10 \mu \mathrm{m}$ thickness from frozen muscle by a cryostat. Collect the purified total RNA using the purification kit of total RNA according to the manufacturer's instructions.

2. Measure the RNA concentration using a spectrophotometer.

3. Combine the required reagents for a RT-PCR reaction in PCR tubes according to Table 1. See Table 2 for primer sequences.

NOTE: The forward primer was 44F for patient NS-03 and $46 \mathrm{~F}$ for patient NS-07. The reverse primer was $54 / 55 \mathrm{R}$ as a default. If non-specific products were evident, $54 \mathrm{R}$ was used as an alternative.

4. Place the PCR tubes containing the mixture in a thermocycler. Run the thermo-cycler according to Table 3.

5. Store PCR product at $4{ }^{\circ} \mathrm{C}$ for short-term storage or -20 ${ }^{\circ} \mathrm{C}$ for long-term storage. 


\section{Microchip electrophoresis and exon skipping calculation}

NOTE: A microchip electrophoresis (MCE) system is often selected to analyze exon skipping efficiency. In this protocol, we describe the steps necessary to analyze the exon skipping efficiency using a MCE system by manufacturer $A$ as well as from manufacturer $B$, hereafter called system $A$ and $B$. During the clinical trial, the exon skipping efficiency was analyzed on system A. However, system A is no longer for sale, and we recommend system $B$ to analyze exon skipping efficiency. We have included protocols for both systems (see section 5.1 for system A and 5.2 for system B). See Table of Materials for information regarding system $A$ and $B$. Furthermore, this step can also be performed with normal agarose gel electrophoresis, but the sensitivity markedly decreases.

\section{Microchip electrophoresis using System A}

NOTE: System A has two types of chips. Here, we describe the steps for the DNA chip.

1. Equilibrate kit reagents (stain buffer, loading buffer, ladder, and gel buffer) to RT, vortex, and spin down.

2. To prepare gel-stain buffer (GS), add $12.5 \mu \mathrm{L}$ of stain buffer to $250 \mu \mathrm{L}$ of gel buffer and vortex for $10 \mathrm{~s}$. Move the solution to a spin filter tube and centrifuge at 2,400 $x g$ for $15 \mathrm{~min}$. Discard the filter.

NOTE: The filtered GS can be stored at $4{ }^{\circ} \mathrm{C}$ for 1 month.

3. If samples are highly concentrated, dilute to approximately $50 \mathrm{ng} / \mu \mathrm{L}$ with TE buffer or DNase-free water.

NOTE: If samples are in a salt concentration $\geq 200$ $\mathrm{mM} \mathrm{KCl}$ (or $\mathrm{NaCl}$ ) and/or $15 \mathrm{mM} \mathrm{MgCl}_{2}$, exchange the buffer.
4. Add $12 \mu \mathrm{L}$ of $\mathrm{GS}$ solution to the gel priming well (highlighted and labelled GS) of the DNA chip and place the chip in the priming station.

NOTE: To avoid air bubbles, insert the pipette tip vertically and to the bottom of the well when dispensing. Dispense slowly to the first stop on the pipette, and do not expel air at the end of the pipetting step.

5. Select C3 mode and press the Start button. After priming is completed, remove the chip.

6. Visually inspect the microchannels for trapped air bubbles or incomplete priming.

7. Load the prepared samples and ladder onto the chip as below.

1. Pipette $9 \mu \mathrm{L}$ of $\mathrm{GS}$ solution into the other $3 \mathrm{GS}$ wells.

2. Pipette $5 \mu \mathrm{L}$ of loading buffer into the $\mathrm{L}$ well and each sample well (1-11).

3. Pipette $1 \mu \mathrm{L}$ of ladder into the $L$ well.

4. Pipette $1 \mu \mathrm{L}$ of DNA sample into each of the 11 sample wells.

5. Pipette $1 \mu \mathrm{L}$ of TE or DNase-free water into any unused wells. The kit quick guide shows a figure of the chip layout.

NOTE: Inspect all wells for air bubbles by holding the chip above a lightly colored background. Dislodge any trapped air bubbles at the bottom of a well with a clean pipette tip or by removing and reloading the solution.

8. Place the chip in the vortex station and press Mix. The vortex station automatically stops after $1 \mathrm{~min}$. 
9. Run the chip in the electrophoresis station within 5 min of loading.

10. Select New Run in the software toolbar. On the New Run screen, select DNA and DNA 1K from the Assay pull-down list.

11. Either select a project folder for the run from the Project pull-down list or create a new project folder by entering a name in the Project field.

12. Enter a name for the run in the Run Prefix field and click Start Run.

13. The instrument beeps when the analysis is complete, and a window opens indicating the end of the run. Select OK and remove the chip from the chip platform.

14. Select File | Export Data to export the data. Select the desired options in the Export dialogue.

15. To clean the electrodes, fill a cleaning chip with 800 $\mu \mathrm{L}$ of deionized water and place it on the chip platform, close the lid, and leave it closed for $1 \mathrm{~min}$. After $1 \mathrm{~min}$, open the lid, remove the cleaning chip, and allow the electrodes to dry for $1 \mathrm{~min}$.

\section{Microchip electrophoresis using system B}

1. Open the operating software and enter the sample information. After entering the information, the software calculates the amount of separating buffer and marker solutions automatically.

2. Prepare the necessary amount of separating buffer calculated by the software. First, prepare $100 x$ nucleic acid gel stain solution by diluting $10,000 x$ solution with the appropriate amount of TE buffer. The 100x solution can be stored at $-20^{\circ} \mathrm{C}$.

3. Mix a suitable amount of $100 x$ nucleic acid gel stain withseparation buffer in the specific tube provided by the company to prepare a $1 \mathrm{x}$ solution. Vortex the solution.

4. Prepare the required amount of marker solution in the tube.

5. Start the probe wash program.

6. Start the microchip wash program when finished.

7. Simultaneously, pipette the samples to a PCR multiplate (96-well, clear) and dilute 4 times with deionized water. The minimum volume the machine can handle is $6 \mu \mathrm{L}$, and the maximum is $30 \mu \mathrm{L}$. We suggest a total volume of $10 \mu \mathrm{L}(2.5 \mu \mathrm{L}$ of sample and $7.5 \mu \mathrm{L}$ of water/TE-buffer).

8. Cover the plates with adhesive PCR sealing foil sheets and spin-down the samples.

9. Set the plate, marker solution, and $1 x$ separating buffer in the machine according to the placement shown by the machine. Push the Start button.

10. When the run is finished, export the result data as a .csv file from the software and calculate the exon skipping efficiency using molar concentration as follows:

Exon skipping efficiency $(\%)=$ (Skipped band $/$ (Skipped band + Non-skipped band) $\times 100$

\section{Complementary DNA (cDNA) sequencing}

\section{Agarose gel preparation}

1. Measure $1.5 \mathrm{~g}$ of agarose powder and mix the powder with $100 \mathrm{~mL}$ of $1 \times$ TAE buffer in a microwavable flask (resulting in a $1.5 \%$ agarose gel).

2. Microwave for $1-3$ min until the agarose is completely dissolved. 
NOTE: Do not overboil the solution, since some of the buffer will evaporate and alter the final agarose percentage of the gel.

3. Let the agarose solution cool to about $50^{\circ} \mathrm{C}$.

4. Add $10 \mu \mathrm{L} 10,000 x$ fluorescent nucleic acid dye to the agarose solution.

5. Pour the agarose into a gel tray with the well comb in place. Leave at RT for 20-30 min until it has completely solidified.

\section{Sequencing}

1. Mix $5 \mu \mathrm{L}$ of RT-PCR product (from step 4.4) and $1 \mu \mathrm{L}$ of $6 x$ loading buffer. Load them into the wells of the $1.5 \%$ agarose gel.

2. Run the gel at $135 \mathrm{~V}$ for $5 \mathrm{~min}$ and $120 \mathrm{~V}$ for $20 \mathrm{~min}$.

3. Visualize the bands using a transilluminator according to the manufacturer's protocol.

4. Excise bands of interest from the gel and use a gel and PCR cleanup kit to retrieve RT-PCR products.

5. Measure the concentration using a spectrophotometer.

NOTE: The purified band can be sent for sequencing at a company or sequencing facility if no Sanger sequencing equipment is available in-house.

6. Prepare the required reagents for a cycle sequencing kit and pipette into a multiplate PCR plate according to Table 4. See Table 2 for primer sequences.

7. Seal the plate with clear adhesive film.

8. Vortex the plate for 2 to $3 \mathrm{~s}$. Centrifuge briefly in a swinging bucket centrifuge so that the contents settle to the bottom of the wells ( 5 to $10 \mathrm{~s}$ ) at $1,000 \times \mathrm{g}$.
NOTE: Air bubbles may be present in the wells, but they do not adversely affect the reaction.

9. Place the mixture in a thermo-cycler and run according to Table 5.

10. Purify the sequencing reactions with plates according to the manufacturer's instructions.

11. Use an DNA analyzer to determine the sequences according to the manufacturer's protocol.

12. Compare the sequence obtained to the patient's expected sequence.

\section{Western blotting}

\section{Sample preparation}

1. Prepare SDS sample buffer (4\% SDS, $4 \mathrm{M}$ urea, 10\% 2-mercaptoethanol, 10\% glycerol, $70 \mathrm{mM}$ Tris$\mathrm{HCl} \mathrm{pH} \mathrm{6.4,} \mathrm{0.001 \%} \mathrm{bromophenol} \mathrm{blue,} \mathrm{and} \mathrm{protease}$ inhibitor).

2. Place around 100 slices of the $10 \mu \mathrm{m}$ muscle sections collected from cryo-sectioning in $150 \mu \mathrm{L}$ of SDS buffer.

3. Briefly homogenize the protein samples on ice for 30 s using a handy micro homogenizer.

4. Centrifuge at $16,500 \times g$ for $15 \mathrm{~min}$, and transfer the supernatant to a fresh tube. Proceed with analysis or store at $-80^{\circ} \mathrm{C}$.

\section{SDS-PAGE for protein concentration measurement}

NOTE: A fluorescent gel stain was used to determine the protein concentration.

1. Determine the normal healthy control sample weight and the concentration using the BCA protein assay kit according to the manufacturer's instructions. 
2. Prepare the samples for gel electrophoresis. Pipette $10 \mu \mathrm{g}$ of total protein of the healthy control and $5 \mu \mathrm{L}$ of the patient samples, $5 \mu \mathrm{L}$ of $4 \mathrm{x}$ sample buffer, $2 \mu \mathrm{L}$ of $10 x$ sample reducing agent. Once these have been mixed, add deionized water to a final volume of $20 \mu \mathrm{L}$ in each sample.

3. Heat samples at $70^{\circ} \mathrm{C}$ for $10 \mathrm{~min}$.

4. Prepare $2,000 \mathrm{~mL}$ of $1 \times$ SDS running buffer using 50 $\mathrm{mL}$ of $40 x$ SDS running buffer. Dilute with $1,950 \mathrm{~mL}$ of deionized water.

NOTE: At this point, $1,000 \mathrm{~mL}$ of running buffer is enough. The remaining $1,000 \mathrm{~mL}$ buffer is used at step 7.3.1.

5. Mix thoroughly and set aside $800 \mathrm{~mL}$ of the $1 x$ SDS running buffer for use in the lower (outer) buffer chamber of the gel box.

6. Immediately prior to electrophoresis, add $500 \mu \mathrm{L}$ of antioxidant buffer to $200 \mathrm{~mL}$ of $1 \times$ SDS running buffer to use in the upper (inner) buffer chamber of the gel box.

7. Prepare for gel electrophoresis by setting up a $3-8 \%$ Tris-acetate gel according to the manufacturer's protocol. Load $20 \mu \mathrm{L}$ of each sample onto the gel.

8. Perform electrophoresis at $150 \mathrm{~V}$ for $75 \mathrm{~min}$.

9. After electrophoresis, place the gel directly into a clean tray containing $50 \mathrm{~mL}$ of fluorescent gel stain solution.

10. Cover the tray, place on a shaker, and agitate without splashing liquid or damaging the gel for $90 \mathrm{~min}$.

11. Transfer the gel into water before imaging in a fluorescence system at $312 \mathrm{~nm}$ illumination with ethidium bromide emission filter to detect fluorescence.

12. Measure the concentrations of the patient samples based on an analytical curve constructed using the normal control lysate with known concentration.

\section{SDS-PAGE for western blotting}

1. Prepare the gel box according to steps 7.2.4-7.2.6.

2. Based on the concentration measurement obtained in step 7.2.11, load $100 \mu \mathrm{g}$ per lane of healthy control lysate and $300 \mu \mathrm{g}$ per lane of the patient sample. Electrophorese using the same settings as step 7.2.7.

\section{Protein transfer}

1. Prepare the transfer buffer. Soak a PVDF membrane for $20 \mathrm{~s}$ in methanol. Move it to the blotting buffer B until use.

2. Soak a extra-thick blotting paper in blotting buffer $A$, $B$, and $C$ for at least 30 min per buffer.

3. After electrophoresis, soak the gel in blotting buffer B for $5 \mathrm{~min}$.

4. Place blotting papers, PVDF membrane, and gel on the semi-dry transfer machine according to the drawing in Figure 1. Roll out air bubbles between the gel and membrane with a roller.

5. Transfer at $2 \mathrm{~mA} / \mathrm{cm}^{2}$ membrane for $1 \mathrm{~h}$ at RT. NOTE: After transferring, it is recommended to perform a total protein stain similar to Ponceau staining to confirm successful transfer of the large molecular weight proteins.

\section{Blocking and antibody staining}

1. Rinse the membrane twice with PBST (1x PBS with $0.1 \%$ Tween 20$)$. 
2. Place the membrane in $1 \%$ blocking agent, and rock gently for $1 \mathrm{~h}$ at RT to block.

3. Incubate the membrane with anti-dystrophin antibody in blocking solution (1:125) and anti-spectrin antibody $(1: 25,000)$ for $1 \mathrm{~h}$ at RT or overnight at $4{ }^{\circ} \mathrm{C}$.

4. Wash the membrane three times for 10 min each with PBST at RT.

5. Incubate the membrane with horseradish peroxidase (HRP)-conjugated secondary anti-mouse/rabbit antibody in PBST (1:100) for $40 \mathrm{~min}$ at RT.

6. Wash the membrane again three times for 10 min with PBST at RT.

\section{Detection}

1. Mix detection solutions $A$ and $B$ in a ratio of $1: 1$. The final volume of detection reagent required is $0.1 \mathrm{~mL} /$ $\mathrm{cm}^{2}$ membrane.

2. Remove the excess PBST from the washed membrane and place it with the protein side up on a sheet of plastic wrap or other suitable clean surfaces. Add the mixed detection reagent onto the membrane and incubate for 5 minutes at RT.

3. Remove the excess detection reagent by holding the membrane gently in forceps and touching the edge against a tissue.

4. Place the blot protein side up on a sample tray. Operate the fluorescent system according to the user documentation. Set machine as follows. Exposure type: Increment, Interval time: $10 \mathrm{~s}$, Sensitivity/ Resolution: high.

NOTE: Measured areas were boxed with a blue rectangle (Figure 4a-b): BG: background; D: dystrophin $427 \mathrm{kDa}$, SL: spectrin beta long isoform
(274 kDa); SS: short isoform (253 kDa). Dystrophin/ spectrin signal ratio was calculated as (D-BG)/[(SL$B G)+(S S-B G)]$. The ratio of normal control was set as $100 \%$. 


\section{Representative Results}

To use RT-PCR to detect exon skipping, primers on either side of the exon that will be skipped were designed and evaluated to yield only specific bands (see Figure 2 for a schematic diagram of exon skipping and primer position). The primers should generate products that can easily be distinguished by size on a MCE system or normal agarose gel electrophoresis if MCE is not available. Figure 3a shows MCE system A gel images of RT-PCR reactions and the sequencing results of the skipped band from patients NS-03 and NS-07 before and after treatment with NS-065/ NCNP-01 in the dose-escalation phase 1 trial. NS-03 and NS-07 harbor deletions that span exons $45-52$ and 48-52, respectively. NS-03 received $5 \mathrm{mg} / \mathrm{kg}$ and NS-07 $20 \mathrm{mg} /$ $\mathrm{kg}$ of NS-065/NCNP-01 weekly for 12 weeks. As expected before treatment, both patients showed no skipping, and only a non-skipped band could be visualized. After 12 weeks of treatment, a clear skipped lower band was visualised for NS-07. However, it was still difficult to detect any skipped product for patient NS-03. The sequencing results showed a concatenation of exons 47 and 54 for NS-07, as well as exons 44 and 54 for NS-03. According to the sequence, these could theoretically produce a functional but shortened dystrophin isoform. To calculate the skipping percentage shown in Figure $\mathbf{3 b}$, the molar concentration for the skipped band was divided by the skipped and un-skipped band. For patient NS-07, the percentage after treatment was $72 \%$, and it was $3.4 \%$ for NS-03. Western blot data (in triplicate) from patients NS-03, NS-07, and a healthy control are shown in Figure 4a. Expectedly, no dystrophin band was detected before treatment. After treatment a band from patient NS-07 was detected with a lower molecular weight compared to healthy control (wild type dystrophin has a molecular weight of $427 \mathrm{kDa}$, and NS-07 dystrophin has a molecular weight of $389 \mathrm{kDa}$ ). Because of the exon deletion and skipping, the dystrophin isoform from patient NS-07 lacked several exons, and it was expected to have a lower molecular weight. Patient NS-03 showed no detectable levels of dystrophin after treatment. In Figure $\mathbf{4 b}$, the amount of dystrophin compared to a healthy control for patient NS-07 is shown. The location where signal intensities were measured for the calculation of dystrophin restoration are indicated with blue squares. The dystrophin spectrin ratio was used to calculate the amount of dystrophin compared to that of the healthy control. To this end, the signal from the dystrophin minus background was divided by the sum of the two spectrin isoforms (long and short) minus background (see step 8.6.5). The ratio for healthy control was set to $100 \%$. The amount of dystrophin compared to the healthy control in patient NS-07 after treatment was $9.1 \%$. However, the percentage could not be calculated for patient NS-03 due to a weak dystrophin band, which similar to those obtained for prior treatment samples for both patients. 


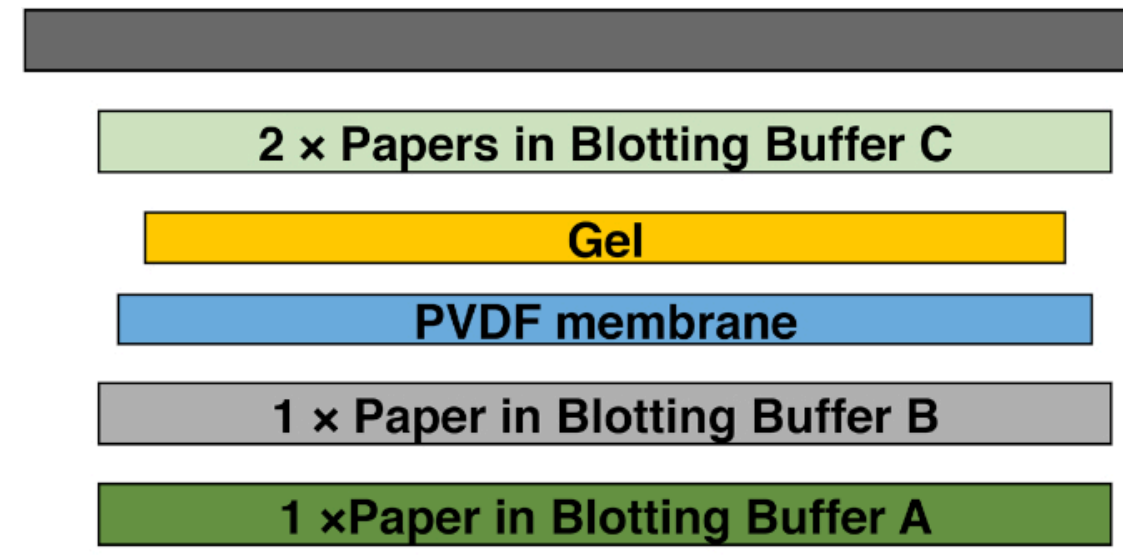

Figure 1: Schematic diagram of the transfer stack for western blot analysis. Assembly of the western blot transfer stack with blotting paper soaked in blotting buffer $A$ in the bottom followed by blotting paper soaked in blotting buffer $B, P V D F$ membrane, the 3-8\% Tris-Acetate Gel and on the top 2 blotting papers soaked in blotting buffer $\mathrm{C}$ is shown. Please click here to view a larger version of this figure.

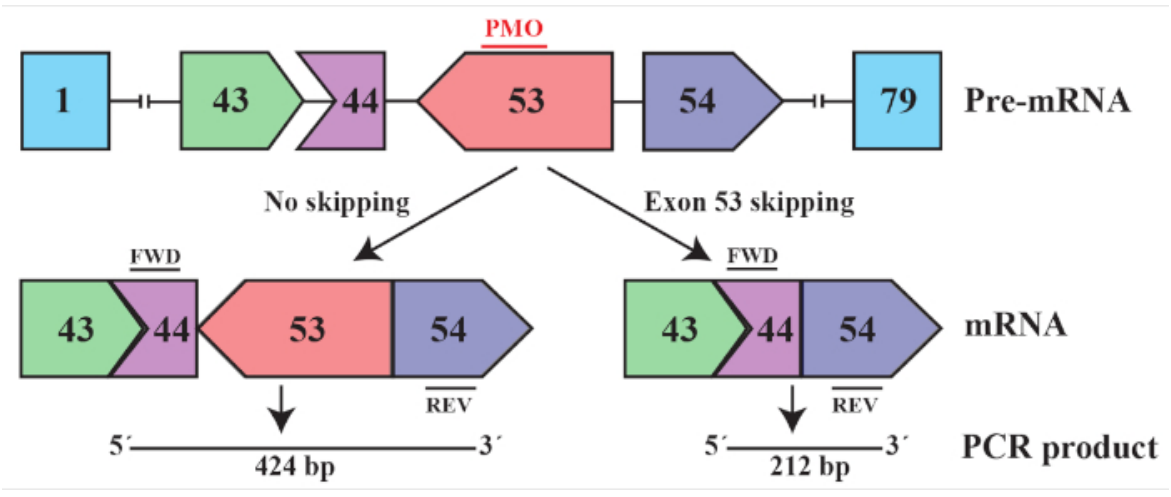

Figure 2: Schematic drawing of exon skipping of exon 53 in a patient with exon 45-52 deletion in the DMD gene. For example, patient NS-03 in the dose-escalation clinical trial had this deletion. If exon 53 is retained, the mRNA will be out of frame since the exon-exon junction between exon 44 and 53 disrupts the reading frame, causing a stop codon in exon 53 . The reading frame is restored when exon 53 is skipped, and a shorter isoform of dystrophin is produced. To detect exon 53skipping by RT-PCR, primers in exon 44 and 54 are used so that the PCR-product between skipped and un-skipped mRNA can easily be detected. FWD: forward primer, REV: reverse primer, PMO: phosphorodiamidate morpholino oligomer. Please click here to view a larger version of this figure. 
a)

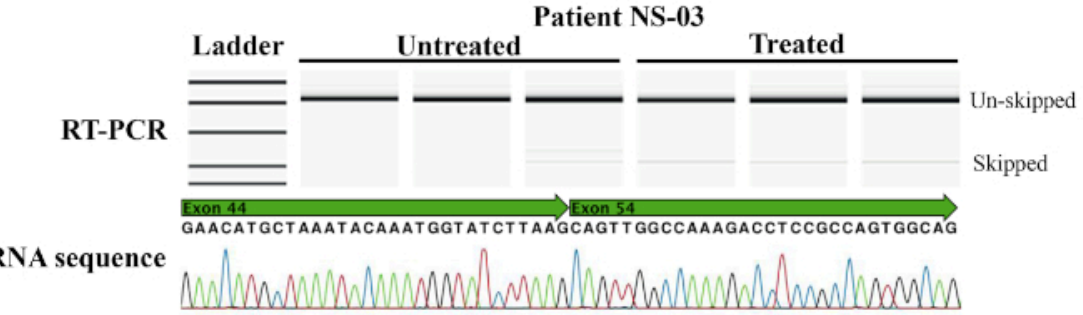

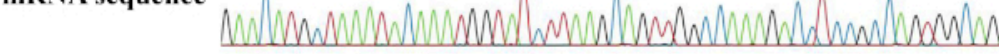

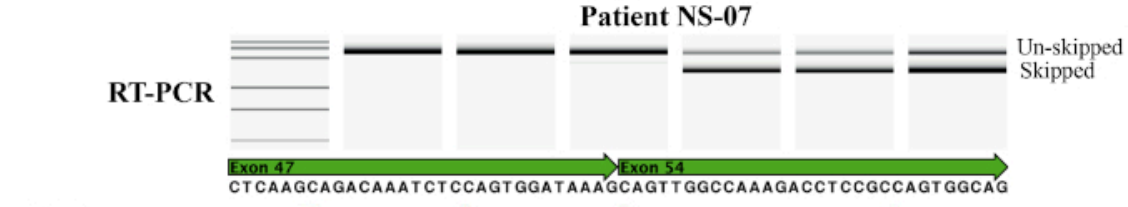

mRNA sequence

CTCAAGCAGACAAATCTCCAGTGGATAAAGCAGITGGCCAAAGACCTCCGCCAGTGGCAO

b)

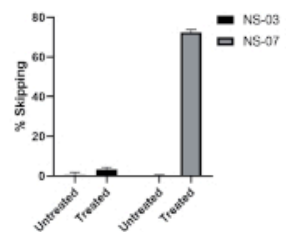

Figure 3: Skipping efficiency for patients NS-03 and NS-07 from tibialis anterior muscle biopsy samples. a) Gel image generated by electrophoresis system A of RT-PCR samples of untreated and treated samples from patients NS-03 and NS-07. Upper panel shows NS-03 and lower panel NS-07 in triplicate. For NS-03, the un-skipped band is 422 bp, and the skipped band is $212 \mathrm{bp}$. For NS-07, the bands are $836 \mathrm{bp}$ and $624 \mathrm{bp}$, respectively. Sequence analysis showed a concatenation of exon 44 and exon 54 for NS-03 and exon 47 to exon 54 for NS-07. b) Skipping efficiency before and after treatment is shown for patients NS-03 and NS-07, calculated from the molar concentration of the two bands provided by system A as skipped band/(skipped band + un-skipped band) x 100. The skipping efficiency for NS-03 was very low after treatment; for NS-07, the skipping efficiency was over $70 \%$. Please click here to view a larger version of this figure. 

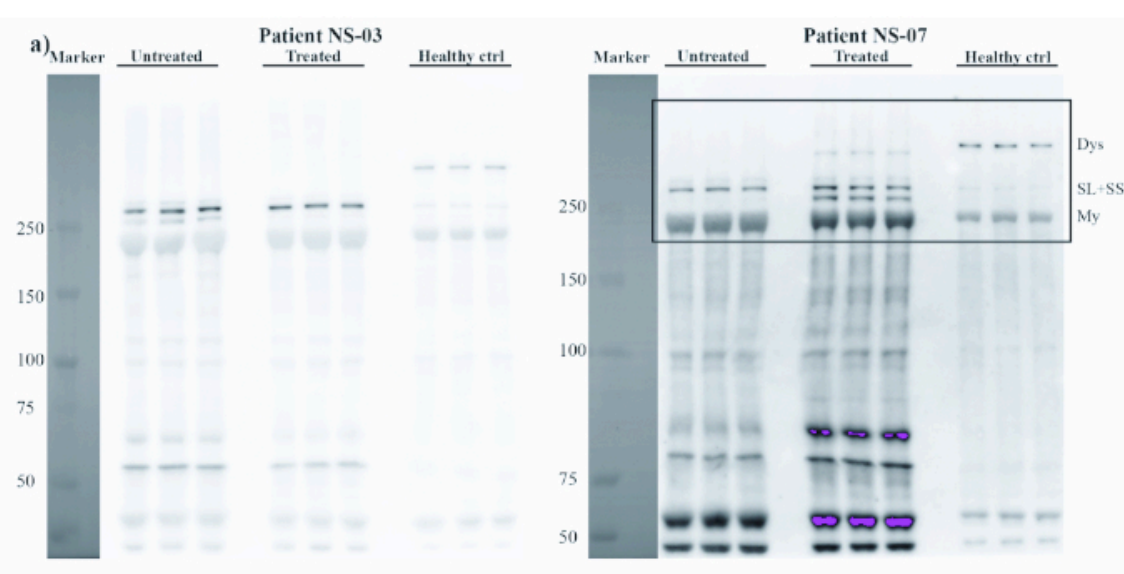

b)
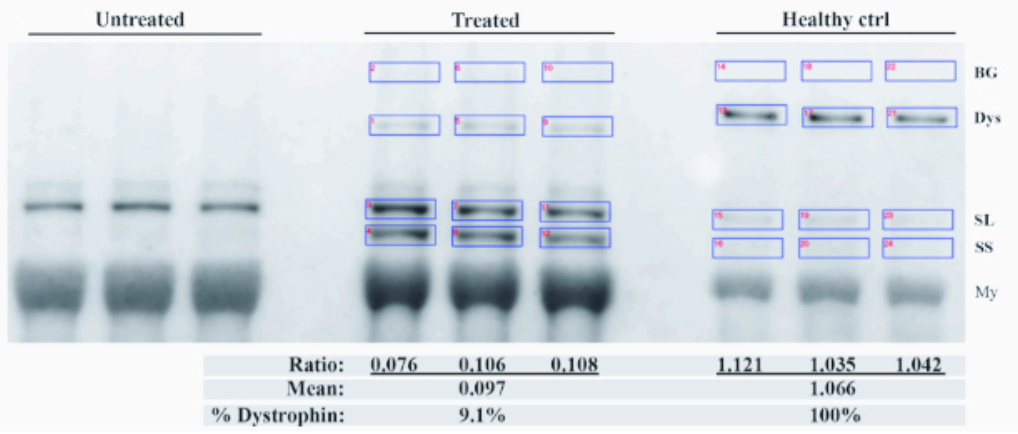

Figure 4: Protein quantification of Dystrophin isoforms before and after exon skipping therapy. a) Protein quantification of dystrophin isoforms before and after exon skipping therapy. Western blot of muscle biopsy from tibialis anterior from patients NS-03 and NS-07 before and after treatment and from healthy control in triplicate. Dystrophin can be seen at $427 \mathrm{kDa}$ for the healthy control and at $389 \mathrm{kDa}$ for NS-07 after treatment. No dystrophin could be detected for either patient before treatment, or neither could any be detected after treatment for patient NS-03. The area in the black box is shown in Figure $\mathbf{4 b}$. To the left in each blot the marker is shown. b) The amount of dystrophin restored after treatment for patient NS-07. Dystrophin restoration was calculated based on the intensities of the bands for dystrophin, background, and the long and short isoform of spectrin according to the formula: (dystrophin - background)/(spectrin L -background) + (spectrin S - background) where the ratio for healthy control is set to $100 \%$. The intensity of each band was measured inside the blue boxes shown in the figure. The dystrophin restoration for NS-07 was $9.1 \%$ after treatment. The dystrophin signal was too weak to measure in the untreated samples. Marker sizes are shown in kilodaltons. Dys: Dystrophin, BG: background, SL: Spectrin long isoform, SS: Spectrin short isoform, My: Myosin type 1. Marker sizes are shown in kilodaltons. Please click here to view a larger version of this figure. 


\begin{tabular}{|c|c|c|}
\hline Solution & Volume/Reaction $(\mu \mathrm{l})$ & Final concentration \\
\hline RNase-free water (provided) & Variable & $1 \mathrm{x}$ \\
\hline 5x QIAGEN OneStep RT-PCR Buffer & 5.0 & $400 \mu \mathrm{M}$ of each dNTP \\
\hline dNTP Mix (containing & 1.0 & $0.6 \mu \mathrm{M}$ \\
\hline 10 mM of each dNTP) & & $0.6 \mu \mathrm{M}$ \\
\hline Forward Primer $(10 \mu \mathrm{M})$ & 1.5 & - \\
\hline Reverse Primer $(10 \mu \mathrm{M})$ & 1.5 & $5-10$ units/reaction \\
\hline QIAGEN OneStep RT-PCR Enzyme Mix & 1.0 & \\
\hline RNase inhibitor (optional) & Variable & \\
\hline Template RNA & $50 \mathrm{ng}$ & \\
\hline Total & 25 & \\
\hline
\end{tabular}

Table 1: RT-PCR reagents. The necessary compounds for one reaction of the RT-PCR.

\begin{tabular}{|c|c|}
\hline Primer & Sequence \\
\hline $44 \mathrm{~F}$ & $5^{\prime}$-CCTGAGAATTGGGAACATGC-3' \\
\hline $46 \mathrm{~F}$ & $5^{\prime}$-AACCTGGAAAAGAGCAGCAA-3' \\
\hline $48 \mathrm{~F}$ & $5^{\prime}$-CCAAGAAGGACCATTTGACG-3' \\
\hline $54 / 55 \mathrm{R}$ & $5^{\prime}$-'TCTCGCTCACTCACCCTTTT-3' \\
\hline $54 \mathrm{R}$ & $5^{\prime}$-GTGGACTTTTCTGGTATCAT-3' \\
\hline
\end{tabular}

Table 2: Primer list. Sequences for the primers used in this study. F: Forward, R: Reverse. 


\begin{tabular}{|c|c|c|c|}
\hline 1 cycle & Reverse transcription & $30 \mathrm{~min}$ & $50^{\circ} \mathrm{C}$ \\
\hline 1 cycle & Initial PCR activation step & $15 \mathrm{~min}$ & $95^{\circ} \mathrm{C}$ \\
\hline \multirow{2}{*}{35 cycles } & Denaturation & $1 \mathrm{~min}$ & $60^{\circ} \mathrm{C}$ \\
\cline { 2 - 4 } & Annealing & $1 \mathrm{~min}$ & $72^{\circ} \mathrm{C}$ \\
\cline { 2 - 4 } & Extension & $1 \mathrm{~min}$ & $72^{\circ} \mathrm{C}$ \\
\hline 1 cycle & Final extension & $7 \mathrm{~min}$ & $4{ }^{\circ} \mathrm{C}$ \\
\hline
\end{tabular}

Table 3: PCR conditioned used. Show PCR conditions for the RT-PCR reaction.

\begin{tabular}{|c|c|}
\hline Solution & Volume/Reaction $(\boldsymbol{\mu l})$ \\
\hline RNase-free water & Variable \\
\hline BigDye Terminator 3.1 Ready Reaction Mix & 3.5 \\
\hline Forward Primer/Reverse Primer $(3.2 \mu \mathrm{M})$ & 2.0 \\
\hline Template RNA & $20 \mathrm{ng}$ \\
\hline Total & 20 \\
\hline
\end{tabular}

Table 4: Reagents necessary for the sequencing reaction. Use either Forward or Reverse primer in the setup, not both at the same time.

\begin{tabular}{|c|c|c|}
\hline 1 cycle & $1 \mathrm{~min}$ & $96{ }^{\circ} \mathrm{C}$ \\
\hline \multirow{2}{*}{25 cycles } & $10 \mathrm{~s}$ & $96{ }^{\circ} \mathrm{C}$ \\
\cline { 2 - 3 } & $5 \mathrm{~s}$ & $50{ }^{\circ} \mathrm{C}$ \\
\cline { 2 - 3 } & $4 \mathrm{~min}$ & $60{ }^{\circ} \mathrm{C}$ \\
\hline Hold & $\infty$ & $4{ }^{\circ} \mathrm{C}$ \\
\hline
\end{tabular}

Table 5: PCR conditions for the Sanger sequencing.

\section{Discussion}

Clinical trials of DMD have produced both successes and failures in the last few years. Both RT-PCR and western blotting are common techniques to assess the skipping efficiency generated by exon-skipping compounds administered to the patients. However, RT-PCR has been 
reported to over-estimate the skipping efficiency compared to digital $\mathrm{PCR}^{15}$. Although this is due to a number of reasons, it is primarily caused by the more efficient amplification of the smaller skipped fragments during PCR cycles. It appears that RT-PCR used in this clinical trial also generated higher skipping efficiencies compared to the protein expression estimated by western blotting. According to the FDA, this is a more reliable way to quantify dystrophin restoration ${ }^{12}$. Hence, caution should be exercised when interpreting RTPCR skipping results; however the samples can still be compared. Samples showing higher skipping efficiencies based on RT-PCR results commonly exhbit higher protein expression levels in western blot analyses.

Since all patients in DMD clinical trials do not have the same deletion pattern, it can be difficult to design primers and probes that are adequately specific to perform digital or qPCR on all samples. Hence, RT-PCR is still a good alternative for a first assessment of the skipping efficiency. Before the clinical trial of NS-065/NCNP-01 commenced, it was tedious to assess skipping efficiency for each patient in vitro since either a muscle or skin biopsy was mandatory to generate patient-specific myoblasts. However, we have recently published a novel technique to generate patientspecific MYOD1-converted, urine-derived cells (UDCs) as a novel DMD muscle cell model ${ }^{16}$. Thus, only urine collected from the patient is required to generate the myoblasts, and no invasive procedure is necessary. We believe that this method can be used to screen different AONs in patientspecific cells. Furthermore, different primers and probes can be tested before the patient starts any clinical trial. This can facilitate the use of $\mathrm{qPCR}$ or digital PCR for exon skipping measurement in DMD clinical trials in the future.
For performing western blot analysis in this clinical trial, a single antibody against dystrophin was used, and only one healthy control was used as a reference sample. Hence, the specificity of the antibody was not validated appropriately. This, together with the fact that the antibody only recognises the C-terminal domain of dystrophin, is a limitation of the protocol. More healthy controls and antibodies directed against different domains of the dystrophin molecule are advisable in the future.

Here, we summarized the protocols that were used in the recent exploratory investigator-initiated, first-in-human trial of NS-065/NCNP-01. NS-065/NCNP-01 is potentially applicable to $10.1 \%$ of patients with DMD.

\section{Disclosures}

None.

\section{Acknowledgments}

We are grateful to Dr Takashi Saito, Dr Tetsuya Nagata, Mr Satoshi Masuda, and Dr Eri Takeshita for scientific discussion.

\section{References}

1. Bushby, K. et al. Diagnosis and management of Duchenne muscular dystrophy, part 2: implementation of multidisciplinary care. Lancet Neurology. 9 (2), 177-189 (2010).

2. Hoffman, E. P., Brown, R. H., Jr., Kunkel, L. M. Dystrophin: the protein product of the Duchenne muscular dystrophy locus. Cell. 51 (6), 919-928 (1987).

3. Mendell, J. R. et al. Evidence-based path to newborn screening for Duchenne muscular dystrophy. Annals of Neurology. 71 (3), 304-313 (2012). 
4. Moat, S. J., Bradley, D. M., Salmon, R., Clarke, A., Hartley, L. Newborn bloodspot screening for Duchenne muscular dystrophy: 21 years experience in Wales (UK). European Journal of Human Genetics. 21 (10), 1049-1053 (2013).

5. Greenberg, C. R. et al. Gene studies in newborn males with Duchenne muscular dystrophy detected by neonatal screening. Lancet. 2 (8608), 425-427 (1988).

6. Forrest, S. M. et al. Further studies of gene deletions that cause Duchenne and Becker muscular dystrophies. Genomics. 2 (2), 109-114 (1988).

7. Monaco, A. P., Bertelson, C. J., Liechti-Gallati, S., Moser, H., Kunkel, L. M. An explanation for the phenotypic differences between patients bearing partial deletions of the DMD locus. Genomics. 2 (1), 90-95 (1988).

8. Flanigan, K. M. et al. Mutational spectrum of DMD mutations in dystrophinopathy patients: application of modern diagnostic techniques to a large cohort. Human Mutation. 30 (12), 1657-1666 (2009).

9. Aoki, Y. et al. In-frame dystrophin following exon 51skipping improves muscle pathology and function in the exon 52-deficient mdx mouse. Molecular Therapy. 18 (11), 1995-2005 (2010).

10. Lu, Q. L. et al. Functional amounts of dystrophin produced by skipping the mutated exon in the $\mathrm{mdx}$ dystrophic mouse. Nature Medicine. 9 (8), 1009-1014 (2003).

11. Yokota, T. et al. Efficacy of systemic morpholino exon-skipping in Duchenne dystrophy dogs. Annals of Neurology. 65 (6), 667-676 (2009).

12. Kesselheim, A. S., Avorn, J. Approving a Problematic Muscular Dystrophy Drug: Implications for FDA Policy.
Journal of the American Medical Association. 316 (22), 2357-2358 (2016).

13. Mendell, J. R. et al. Eteplirsen for the treatment of Duchenne muscular dystrophy. Annals of Neurology. 74 (5), 637-647 (2013).

14. Komaki, H. et al. Systemic administration of the antisense oligonucleotide NS-065/NCNP-01 for skipping of exon 53 in patients with Duchenne muscular dystrophy. Science Translational Medicine. 10 (437) (2018).

15. Verheul, R. C., van Deutekom, J. C., Datson, N. A. Digital Droplet PCR for the Absolute Quantification of Exon Skipping Induced by Antisense Oligonucleotides in (Pre-)Clinical Development for Duchenne Muscular Dystrophy. PLoS One. 11 (9), e0162467 (2016).

16. Takizawa, H. et al. Modelling Duchenne muscular dystrophy in MYOD1-converted urine-derived cells treated with 3-deazaneplanocin A hydrochloride. Scientific Reports. 9 (1), 3807 (2019). 